\title{
Pattern Spectra from Different Component Trees for Estimating Soil Size Distribution
}

\author{
Petra Bosilj ${ }^{1,2}[0000-0001-9640-9828]$, Iain Gould 2 [0000-0001-5315-2241], Tom \\ Duckett $^{1 \text { [0000-0003-2971-7905], and Grzegorz Cielniak }}{ }^{1[0000-0002-6299-8465]}$ \\ 1 Lincoln Centre for Autonomous Systems, School of Computer Science, University \\ of Lincoln, Brayford Campus, LN6 7TS Lincoln, UK \\ 2 Lincoln Institute for Agri-food Technology, University of Lincoln, Riseholme Park, \\ LN2 2LG Lincoln, UK \\ \{pbosilj,igould, tduckett,gcielniak\}@lincoln.ac.uk
}

\begin{abstract}
We study the pattern spectra in context of soil structure analysis. Good soil structure is vital for sustainable crop growth. Accurate and fast measuring methods can contribute greatly to soil management decisions. However, the current in-field approaches contain a degree of subjectivity, while obtaining quantifiable results through laboratory techniques typically involves sieving the soil which is labour- and timeintensive. We aim to replace this physical sieving process through image analysis, and investigate the effectiveness of pattern spectra to capture the size distribution of the soil aggregates. We calculate the pattern spectra from partitioning hierarchies in addition to the traditional max-tree. The study is posed as an image retrieval problem, and confirms the ability of pattern spectra and suitability of different partitioning trees to re-identify soil samples in different arrangements and scales.
\end{abstract}

Keywords: pattern spectra $\cdot$ inclusion trees $\cdot$ partitioning trees $\cdot$ soil structure

\section{Introduction and Motivation}

Soil structure concerns the arrangement of soil aggregates to provide an environment to facilitate good access to water, air and nutrients, and a suitable medium for root development [6]. Good soil structure is fundamental to sustainable crop growth, and can contribute to reducing the environmental impact of agriculture. Hence, robust and accurate methods to measure soil structure are important tools for informing soil management decisions.

A range of methods is available to assess soil structure, from in-field visual investigations [19,13], to lab-based assessments of soil aggregates and structure [1], both with their individual merits and drawbacks. Field-based methods can provide a rapid 'by eye' assessment by field practitioners, but as such rely on some degree of subjectivity, limiting the reliability of comparison between different users. Conversely, laboratory techniques produce a quantifiable result for comparison of soil structure, but these methods tend to be time-consuming and 
labour-intensive. Developments in image analysis of soil aggregate distribution could provide a solution to some of these limitations, either by providing quantifiable, and comparable, units to in-field visual assessments, or by speeding up the laboratory process through replacement of manual sieving.

The laboratory techniques quantify the soil structure in terms of ratios of soil aggregates of certain sizes in the soil. In order to separate aggregates of different sizes, a stack of sieves of decreasing weight sizes is used. The residue in each sieve is then measured in terms of aggregate volume or mass, and used to categorise the soil into 5 categories ranging from friable (Sq1) to very compact (Sq5) according to the VESS (Visual Evaluation of Soil Structure) methodology. This physical process closely matches the algorithmic process of pattern spectra [15] where the image is filtered with a succession of openings of increasing sizes, often described as sieving. They can be calculated from a granulometry [16, 5], where efficiency is achieved using a hierarchy such as a max-tree [22]. The amounts of image content removed by each attribute filter are used as global [25] or region descriptors [3]. We investigate the pattern spectra calculated on both inclusion trees, which are extrema-oriented, and partitioning trees, which capture intermediate level regions, and confirm their suitability for characterising soil structure through evaluation on an image retrieval problem.

In the next section, we outline the related literature on applying image processing and morphological techniques to calculate particle size distribution in soil and similar materials. The methodology for calculating the pattern spectra, as well as the different component trees used, are explained in Sec. 3. The dataset, collected by the authors for the study, is presented in Sec. 4. We elaborate our experiments and results in Sec. 5, and conclude in Sec. 6, outlining future directions of interest.

\section{Related Work}

Some of the earliest image processing techniques applied to estimation of aggregate size distribution focused on segmenting images of non-overlapping coarse aggregates $(3 \mathrm{~mm}$ to $63 \mathrm{~mm}$ ) [18]. Several segmentation-based techniques for working with overlapping particles of coarse-grained sediments, including those based on top-hat and watershed segmentation, are described in [12]. Size distribution of overlapping particles of coarse sands and gravel $(0.7 \mathrm{~mm}$ to $20 \mathrm{~mm}$ ) has also been analysed through statistical image properties [7], and correlated to sample distribution through regression over the images in a "look-up catalogue". In summary, these approaches are limited to cases of no to little particle overlap and samples comprising large aggregates, with further drawbacks including the reliance on segmenting the image into individual particles or a catalogue.

Granulometry [16], followed by pattern spectra [15], is one of the earliest morphological operations, and was developed as a tool for scale (size) and shape analysis of image content, with some of the earliest applications in petrography (studying the grain structure of rocks). Pattern spectra through opening and closing with reconstruction were used for the granulometric analysis of estuarine 
and marine sediments [11], as well as soil section images [10] (also including spectra based on area openings), however both applications focus on samples with mostly non-overlapping aggregates.

Pattern spectra based on area openings calculated on a max-tree were used to produce accurate grain size distributions of sands (smallest reported particle size $0.06 \mathrm{~mm}$ ) [20]. Image granulometry is also discussed in the context of estimating the size distribution of stone fragments [21]. A recent study compares pattern spectra based on different structuring elements as well as area openings, closings and their combination for assessment of grain size for fine and coarse aggregates of sands and pebbles $(0.125 \mathrm{~mm}$ to $16 \mathrm{~mm})$ [2], obtaining the best results through attribute morphology. The mean grain size was estimated through regression on the training samples, by assuming a quadratic relation between measured grain size and image granulometry. The image granulometry was also related to the measured weight distribution of the samples. However, this work was validated on prepared samples with predetermined uniform grain size distribution, while processing partial images of very large samples $(170 \mathrm{~kg}$ of soil).

We study the effectiveness of size pattern spectra in distinguishing soils in terms of their structure, i.e. soil aggregate distribution, posed as an image retrieval problem. The sample sizes used in our experiments are much smaller than those used in previous work (400 g samples, cf. Sec.4 compared to $170 \mathrm{~kg}$ [2]), aiming at the eventual application of the technique to freshly dug samples on site. The image acquisition setup allows us to examine the effectiveness of this approach for cases of completely to partially overlapping or touching aggregates, which have not been examined before in the same study. We compare using 5 different component trees for pattern spectra calculation, including both inclusion $[22,17]$ and partitioning hierarchies $[23,24]$. We propose to calculate the pattern spectra on partitioning trees by relying on the filtering rules outlined in [4]. Additionally, we investigate the influence of three different measures for defining a granulometry outlined in [8].

\section{Methodology}

In this section, we recall the concepts of granulometry and pattern spectra as well as attribute filtering, followed by a short overview of the component trees used in the study.

\subsection{Granulometries and Pattern Spectra}

Both granulometries [16] and pattern spectra [15] rely on openings and closings, and are used to capture the information on the distribution of image component sizes (later extended to shape $[26,25])$. While granulometries can be seen as size distributions of images, pattern spectra are the corresponding size histograms. When implemented through attribute filters, they interact directly with connected components of the image instead of pixels. 


\begin{tabular}{|l|l|l|l|l|l|l|}
\hline 3 & 3 & 3 & 3 & 3 & 3 & 3 \\
\hline 3 & 2 & 2 & 2 & 2 & 2 & 3 \\
\hline 3 & 2 & 1 & 2 & 4 & 2 & 3 \\
\hline 3 & 2 & 2 & 2 & 2 & 2 & 3 \\
\hline 3 & 3 & 3 & 3 & 3 & 3 & 3 \\
\hline 3 & 0 & 0 & 0 & 3 & 3 & 3 \\
\hline 3 & 3 & 3 & 3 & 3 & 3 & 3 \\
\hline
\end{tabular}

(a)

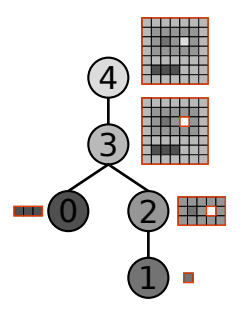

(b)

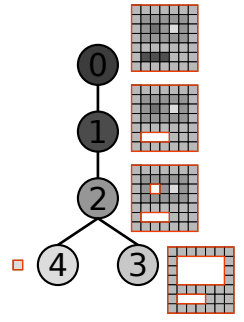

(c)

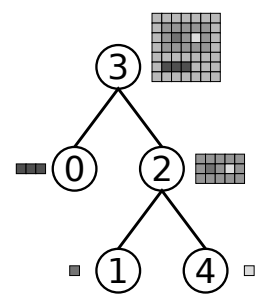

(d)

Fig. 1. The three different inclusion trees of a toy image (a). The min-tree is displayed in (b), while its dual max-tree is shown in (c). The self-dual tree of shapes is shown in (d). The (gray) levels of the nodes are displayed in the nodes, and the corresponding regions are shown beside the nodes.

More formally, we define a grayscale image as $f: E \rightarrow \mathbb{Z}, E \subseteq \mathbb{Z}^{2}$, and the sets of images obtained by thresholding the image $f$ at all possible values of its pixels, called its upper-level sets, as $\mathcal{L}^{k}=\{f \geq k\}$ with $k \in \mathbb{Z}$ (resp. lower-level sets $\mathcal{L}_{k}=\{f \leq k\}$ ). These sets are composed of their connected components, typically based on 4- or 8-connectivity, referred to also as peak components $\mathcal{P}$.

Attribute filtering is an operation applied to these peak components by evaluating a logical predicate on all the peak components by comparing an attribute $\alpha$ (e.g. area) with a threshold $t$ (e.g. $300 \mathrm{px}$ ). An attribute, such as area, is increasing if, for two nested peak components $\mathcal{P}_{A} \subseteq \mathcal{P}_{B}$, its value is always greater for the larger region. The peak components not satisfying the logical predicate are removed from the input image. Filtering with an increasing attribute results in an attribute opening $\Gamma_{t}$ (as it satisfies all the properties of an opening, i.e the anti-extensivity, increasingness and idempotence).

A series of such openings with increasing size $\left\{\Gamma_{t_{i}}\right\}, t_{i+1}>t_{i}$ is called a size granulometry $[16,5]$. More components are removed from the image in every successive opening and the process can be seen as consecutively sieving the image with increasing mesh sizes. To quantify a granulometry $\left\{\Gamma_{t_{i}}\right\}$, the amount of detail remaining in the image is noted. A pattern spectrum is calculated from the granulometry by instead measuring the amount of detail removed between each successive pair of openings.

\subsection{Component Trees}

In attribute morphology, the typical way to interact with connected components of the image is to define them through a component tree, a hierarchical image representation. We distinguish inclusion hierarchies (examples in Fig. 1) comprising partial image partitions as cross-sections, which are typically extremaoriented, and partitioning hierarchies (cf. Fig. 2) with nested image partitions as cross-sections, which are better at representing regions at intermediate values.

The min and max-trees are dual hierarchies belonging to the class of inclusion trees and modelling the inclusion between the peak components of the lower and 


\begin{tabular}{|l|l|l|l|l|}
\hline 0 & 0 & 3 & 3 & 5 \\
\hline 1 & 2 & 4 & 3 & 5 \\
\hline 4 & 1 & 2 & 5 & 2 \\
\hline 4 & 5 & 2 & 0 & 3 \\
\hline 2 & 3 & 0 & 1 & 0 \\
\hline
\end{tabular}

(a)

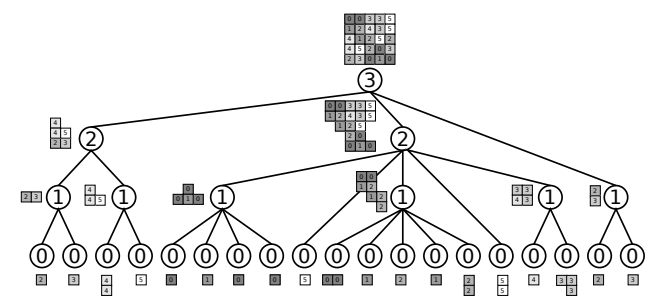

(b)

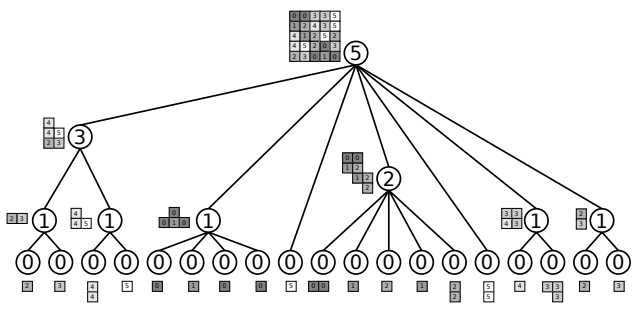

(c)

Fig. 2. For the toy image in (a), the $\alpha$-tree is displayed in (b), while the constrained hierarchy $(\omega)$-tree is shown in (c). The $\alpha$ (resp. $(\omega)$ ) levels are displayed in the nodes and indicated by their height, with regions displayed besides the nodes.

upper level sets of the image, and are well-suited for representing dark and bright components of the image, respectively. As such, they are the ones typically used for calculating the attribute openings and closings defining a pattern spectrum. Examples are shown in Fig. 1(b) and (c).

The tree of shapes (ToS) [17] unifies the representation of bright and dark image structures, producing a single self-dual image representation, treating bright and dark components equally based on their absolute contrast with their background. It comprises all the peak components of both upper and lower level sets with their holes filled, which also form an inclusion hierarchy. An example of a tree of shapes is shown in Fig. 1(d).

The $\alpha$-tree is a partitioning tree based on the local range of its components $[23,24]$ (also sometimes referred to as quasi-flat zone hierarchy [9]). The finest segmentation contained in the leaves of the tree comprises connected components of maximum extent of pixels at the same grey level, which are then merged according to the local neighbour similarity. As such, this hierarchy is capable of representing both bright, dark and intermediate level regions. However, due to the locality of the criterion used, the grey level variations within regions tend to be much higher than $\alpha$ when the grey levels in the image increase and decrease gradually, called the chaining effect [24]. An example of the hierarchy is shown in Fig. 2(b), with the chaining effect observable for $\alpha=2$.

The most notable constrained connectivity hierarchy designed to deal with the chaining effect is the $(\omega)$-tree [24], which rearranges the regions of the $\alpha$ tree according to their global intensity range, removing some of the regions but providing better grouping per level than just a local measure (cf. Fig.2(c)). 
Table 1. The different scale settings used.

\begin{tabular}{c||c|c|c}
\hline Scale & close & middle & far \\
\hline \hline Area $\left[\mathrm{cm}^{2}\right]$ & $15 \times 15$ & $20 \times 20$ & $25 \times 25$ \\
Camera height $[\mathrm{cm}]$ & 60 & 78 & 94 \\
Image resolution $\left[\mathrm{px} \mathrm{mm}{ }^{-1}\right]$ & 16.7 & 12.5 & 10
\end{tabular}

A fast implementation of granulometry and pattern spectra has been made possible by their implementation on max-tree and min-tree image hierarchies [ 5 , 22 , as attribute filtering can be achieved through removing nodes or branches of the tree. The attribute of interest is calculated for all the regions during tree construction, followed by determining the first opening from the sequence $\left\{\Gamma_{t_{i}}\right\}$ interacting with each region which corresponds to the bin $i$ to which the region will contribute (cf. [25] for a more detailed description of this process). Different measures $\mathcal{M}$ can be used to describe the amount of detail contained in the nodes removed between consecutive attribute openings (i.e. remaining detail for the granulometry calculation), the most conventional being the area of the removed regions (in terms of number of pixels). We evaluate the pattern spectra calculated additionally on the other two measures proposed in the context of attribute profiles [8], the number of changed regions, as well as the sum of gray level values. To define attribute filters on the partitioning trees for granulometry and pattern spectra calculation, we follow the procedures outlined in [4].

\section{Dataset Description}

In this section, we describe the dataset collected by the authors to study the application of image processing techniques to soil structure assessment. Prior to taking images, we broke apart the soil structure into constituent aggregates in accordance with the VESS methodology [13]. To analyse a range of soils, we selected soils of different texture and structure. Each sample weighted approximately $400 \mathrm{~g}$, with precise sample weight and aggregate size distribution noted down following the sieving process. Soil A was a calcareous sandy clay loam with a sub-angular to medium granular structure and occasional small stones (VESS category - Sq2). Soil B was a stone-free silt loam, sieved to $5 \mathrm{~mm}$ in order to create a uniform soil structure for the analysis (VESS category - Sq1). Soil C was a clay loam with a sub-rounded to medium granular structure (VESS category Sq3). Soil D was a fine granular to single grained sandy silt loam with occasional stones (structureless). Examples of the soils are given in Table 2.

To create a uniform moisture content across samples, all samples were dried in an oven at $105{ }^{\circ} \mathrm{C}$ for 24 hours, a standard procedure in soil science. Additionally, we have also collected images of the soils $\mathrm{C}$ and $\mathrm{D}$ as-dug, i.e. before drying them in the oven. Square surfaces of three different sizes (cf. Table 1) were drawn on a white tray (shown in Fig. 3(a)), the soils placed in the tray and then manipulated with brushes to fit the marked surface. This setup allowed us to produce images at different pixel resolutions as well as examine the influence of 
Table 2. Examples of all the soil samples A - D at the far scale (area $25 \times 25 \mathrm{~cm}^{2}$ ).

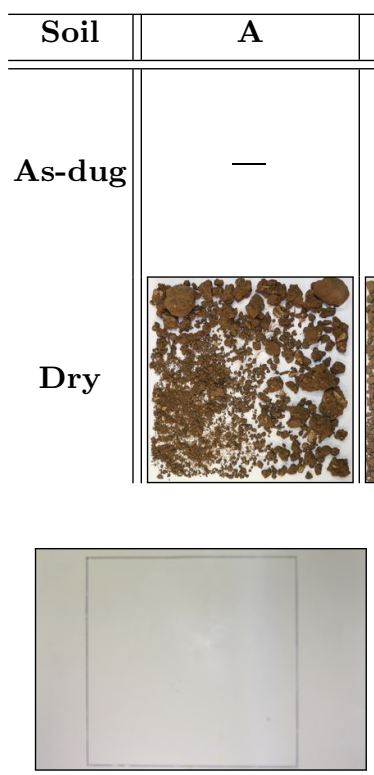

(a)

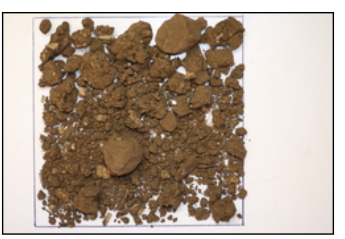

(b)

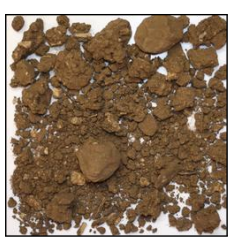

(c)

Fig. 3. The image acquisition setup: (a) the middle $\left(20 \times 20 \mathrm{~cm}^{2}\right)$ square marked on the tray, (b) one of the original images of soil A, (c) the final image obtained by applying the rectifying homography.

the visible background surface in the sample images. The images were taken with a Canon EOS 40D camera, which was placed at a fixed height for a top-down view of the samples and produced images of size $3888 \mathrm{px} \times 2592 \mathrm{px}$, cf. Fig. 3(b). The fixed height was empirically determined to allow for maximal pixel resolution for each of the three marked surface sizes (details in Table 1). Finally, after taking the images, the corners of the marked square were taken as markers for applying a homography to the images to produce a perfect top-down image as well as discard the parts of the image not picturing the sample. The resulting images (example in Fig. 3(c)) are of size 2500 px $\times 2500$ px. The soils were fitted into each of the marked surfaces twice, resulting in two different arrangements of each soil at each scale for a total of 36 images in the dataset.

\section{$5 \quad$ Experiments and Results}

We design our experiments as an image retrieval problem, and assign two sets of ground truth matches (cf. Fig.4) to examine the pattern spectra performance in two distinct cases. In the first case (image pairs marked in blue on Fig. 4 considered correct matches), we examine the ability of pattern spectra to describe the soil samples and allow for their re-identification after rearranging the samples at the same scale only. In the second case (blue and green matches in Fig. 4), we 


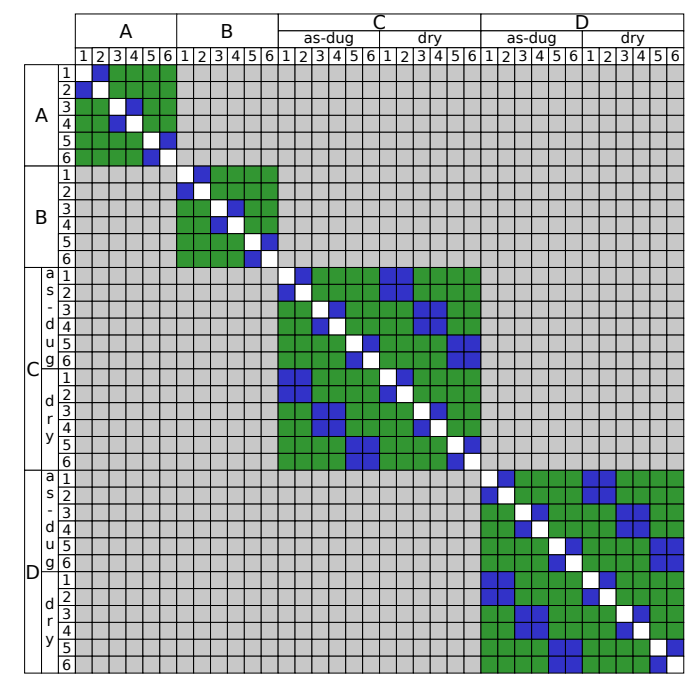

Fig. 4. The ground truth table for both image retrieval setups. Blue - same scale, green - all scales, grey - false matches, white - samples themselves.

Table 3. The bin limits used (with estimated area in pixels).

\begin{tabular}{c|c||c|c|c|c|c|c|c}
\hline \multicolumn{2}{l|}{ mesh size [mm] } & 0.212 & 2 & 3.15 & 5.0 & 9.5 & 25 & 50 \\
\hline \hline \multirow{2}{*}{ close e } & side [px] & 4 & 34 & 53 & 84 & 159 & 417 & 834 \\
& area [px] & 13 & 1112 & 3757 & 6945 & 25070 & 173612 & 694445 \\
\hline \multirow{2}{*}{ middle } & side [px] & 3 & 25 & 40 & 63 & 119 & 313 & 625 \\
& area [px] & 8 & 625 & 1551 & 3907 & 14102 & 97657 & 390626 \\
\hline \multirow{2}{*}{ far } & side [px] & 3 & 20 & 32 & 50 & 95 & 250 & 500 \\
& area [px] & 5 & 400 & 993 & 2500 & 9025 & 62500 & 250000
\end{tabular}

consider re-identifying the samples at all scales present in the dataset. Posing this as an image retrieval problem has two advantages over the alternative setups. Firstly, we only need to change the evaluation metric (i.e. the ground truth), but not the retrieval setup, to examine these two distinct cases, while we would have had to train two different classifiers (one with 4 and one with 24 classes) had we posed it as a classification problem. Secondly, since our final goal is directly relating the pattern spectra to the physically measured soil size distributions, we want to avoid the aggregation step often present in classification and work with the descriptors directly.

The bin thresholds are chosen in two different ways and the performance of the resulting descriptors compared. The upper limit for the largest bin is set to the largest expected particle size of $50 \mathrm{~mm}$ (all the aggregates of all the samples A-D passed through a $50 \times 50 \mathrm{~mm}^{2}$ sieve). We firstly test the logarithmic binning, which is commonly used with pattern spectra descriptors [3]. Secondly, we also use the physical dimensions of each of the sieve meshes to calculate the area of grid openings in pixels for each of the scales (shown in Table 3), and use 
those as bin limits. The number of bins in the logarithmic binning is set to the same number of bins determined by the physical sieves used, $b=7$.

We use 3 different inclusion trees (min and max-tree, tree of shapes) and 2 different partitioning trees $(\alpha$ and $(\omega)$-tree) for pattern spectra calculation (outlined in Sec. 3.2), as well as the sum of the histograms obtained from the minand max-trees (corresponding bins are summed to obtain a new histogram of the same length; denoted as min+max). Each image is described by its associated pattern spectrum, normalised so that the sum of all histogram values equals 1.

After calculating a pattern spectrum for every image, query results for an image are obtained by ranking the other database images according to descriptor similarity. We quantify our retrieval performance in terms of mean Average Precision (mAP), a measure designed specifically to evaluate ranked retrieval results [14] and provide a single measure of quality across all recall levels of a system for a set of multiple queries. For a single query image $q$, we can calculate precision and recall considering only the first $m$ returned images in an unordered fashion. Precision at $m$ is the ratio between the number of relevant images in the set of results and the total number of images retrieved at that point, $m$, while the recall at $m$ is the ratio between the number of relevant images in the results and the total number of relevant images for that query. AP is the Average Precision of a query, and is equivalent to averaging the precision values obtained for the ordered retrieval results, after retrieving each new relevant result:

$$
\begin{aligned}
\mathrm{AP} & =\sum_{m=1}^{K} \operatorname{precision}(m) \times \Delta \operatorname{recall}(m) \\
& =\frac{\sum_{m=1}^{K} \operatorname{precision}(m) \times \operatorname{relevant}(m)}{\text { relevantTotal }},
\end{aligned}
$$

where relevant $(m)$ is an indicator variable indicating if the $m$-th retrieved image is relevant. The mAP is calculated as the mean value of the AP for all the queries.

While we evaluated the retrieval similarity for 5 different histogram similarity metric, we have found very similar trends between the results obtained by $L_{1}$ and $L_{2}$, as well as between the results obtained by $\chi^{2}$ and Bhattacharyya, while the cosine distance underperformed in comparison to the other 4 . Thus, the final results are presented using $L_{1}$ and $\chi^{2}$ distances only.

The results confirm the suitability of pattern spectra for the analysis of soil structure, with achieving the mAP of $66.9 \%$ considering the samples at the same scales only (cf. Table 4), and $78.2 \%$ when considering all the scales (shown in Table 5). Out of the three measures of image content which were used, $\mathcal{M}_{\text {area }}$ has the most consistency, performing well in both cases, with $\mathcal{M}_{\text {volume slightly }}$ underperforming in the single scale experiments and $\mathcal{M}_{\text {count }}$ resulting in a large performance drop of $30 \%$ in the experiments all the scales. We also notice a better performance of the $L_{1}$ over the $\chi^{2}$ distance for $\mathcal{M}_{\text {volume }}$ and $\mathcal{M}_{\text {area }}$ which are measures of the region size, while the $\chi^{2}$ is preferred for $\mathcal{M}_{\text {count }}$ where the regions are simply counted. Additionally, we can observe that using the sieve mesh sizes to define the bin limits improves the retrieval performance compared 
Table 4. Performance of the retrieval system for the same scale ground truth. All results are expressed in terms of $\mathrm{mAP}$ as percentages [\%].

\begin{tabular}{c||c|c|c|c|c|c||c|c|c|c|c|c}
\hline \multicolumn{1}{c||}{} & \multicolumn{5}{c||}{ sieve bins } & \multicolumn{5}{c}{ logarithmic bins } \\
\hline content measure & $\mathcal{M}_{\text {volume }}$ & \multicolumn{2}{c|}{$\mathcal{M}_{\text {area }}$} & \multicolumn{2}{c|}{$\mathcal{M}_{\text {count }}$} & $\mathcal{M}_{\text {volume }}$ & $\mathcal{M}_{\text {area }}$ & \multicolumn{2}{c}{$\mathcal{M}_{\text {count }}$} \\
\hline distance & $L_{1}$ & $\chi^{2}$ & $L_{1}$ & $\chi^{2}$ & $L_{1}$ & $\chi^{2}$ & $L_{1}$ & $\chi^{2}$ & $L_{1}$ & $\chi^{2}$ & $L_{1}$ & $\chi^{2}$ \\
\hline \hline -tree & $\mathbf{5 8 . 1}$ & 47.6 & 63.3 & 56.2 & 62.4 & 64.8 & 40.8 & 37.5 & 52.0 & 51.5 & 31.5 & 40.8 \\
$(\omega)$-tree & 56.7 & 45.4 & $\mathbf{6 5 . 3}$ & 55.1 & 59.4 & $\mathbf{6 6 . 9}$ & 27.3 & 27.5 & $\mathbf{5 2 . 2}$ & 50.2 & 41.9 & $\mathbf{6 4 . 7}$ \\
min-tree & 45.8 & 45.6 & 46.5 & 44.3 & 42.7 & 54.4 & $\mathbf{4 2 . 6}$ & 42.1 & 43.9 & 42.4 & 35.4 & 39.8 \\
max-tree & 26.7 & 26.9 & 32.1 & 33.1 & 62.8 & 66.5 & 32.2 & 32.1 & 40.0 & 42.2 & 45.3 & 45.3 \\
min + max & 29.7 & 30.2 & 32.4 & 33.3 & 49.7 & 62.7 & 32.0 & 31.4 & 39.7 & 39.2 & 38.8 & 42.4 \\
ToS & 45.5 & 42.6 & 46.8 & 45.4 & 46.7 & 59.2 & 39.9 & 39.4 & 40.7 & 40.9 & 38.0 & 41.2 \\
\hline \hline
\end{tabular}

Table 5. Performance of the retrieval system for when all scales are considered in the ground truth. All results are expressed in terms of mAP as percentages [\%].

\begin{tabular}{c||c|c|c|c|c|c||c|c|c|c|c|c}
\hline \multicolumn{1}{c||}{} & \multicolumn{5}{c||}{ sieve bins } & \multicolumn{5}{c}{ logarithmic bins } \\
\hline content measure & $\mathcal{M}_{\text {volume }}$ & \multicolumn{2}{c|}{$\mathcal{M}_{\text {area }}$} & \multicolumn{2}{c}{$\mathcal{M}_{\text {count }}$} & $\mathcal{M}_{\text {volume }}$ & $\mathcal{M}_{\text {area }}$ & \multicolumn{2}{c}{$\mathcal{M}_{\text {count }}$} \\
\hline distance & $L_{1}$ & $\chi^{2}$ & $L_{1}$ & $\chi^{2}$ & $L_{1}$ & $\chi^{2}$ & $L_{1}$ & $\chi^{2}$ & $L_{1}$ & $\chi^{2}$ & $L_{1}$ & $\chi^{2}$ \\
\hline \hline$\alpha$-tree & 70.6 & 68.7 & 60.9 & 66.3 & 35.2 & 35.7 & 69.5 & 69.4 & 72.4 & 71.3 & 41.9 & 43.9 \\
$(\omega)$-tree & 70.1 & 69.2 & 61.2 & 66.1 & 34.5 & 36.4 & 57.6 & 56.9 & 72.1 & 70.9 & 41.1 & 46.7 \\
min-tree & 70.8 & 70.8 & 69.9 & 70.07 & 36.9 & 42.3 & 69.5 & 68.5 & 68.3 & 67.5 & 54.0 & $\mathbf{5 7 . 7}$ \\
max-tree & 71.2 & 71.1 & 75.6 & 75.3 & 38.5 & $\mathbf{4 5 . 1}$ & 67.3 & 68.9 & 71.9 & 72.8 & 53.3 & 56.4 \\
min + max & $\mathbf{7 5 . 4}$ & 75.1 & $\mathbf{7 8 . 2}$ & 77.2 & 37.2 & 44.3 & 72.9 & 73.3 & $\mathbf{7 5 . 9}$ & 75.6 & 53.2 & 56.6 \\
ToS & 73.4 & 73.6 & 72.0 & 72.6 & 35.0 & 43.5 & $\mathbf{7 3 . 5}$ & 73.2 & 72.7 & 72.5 & 53.7 & 56.1 \\
\hline \hline
\end{tabular}

to the traditional logarithmic binning (except in the case of $\mathcal{M}_{\text {count }}$ across all scales, where performance is already low).

Finally, we can also observe that the partitioning trees greatly improve the retrieval results of soil images at the same scale for all the metrics, while the inclusion trees perform well only using the $\mathcal{M}_{\text {count }}$ metric. This was expected as we have observed the openings based on inclusion trees do not interact with aggregates positioned at the border with the background (cf. Fig. 5), which are correctly represented through partitioning trees and their ability to hold regions of intermediate level. This does not hold when considering all the scales, where the best performance was achieved using either the combination of min and maxtrees, or the tree of shapes. The overall results reaffirm the claim that including dual information improves the descriptive power of pattern spectra [2], either by using the min and the max-tree together or one of the self-dual representations. The drop in performance of the partitioning trees compared to inclusion trees at all scales, which we plan to further investigate, is however not too severe, so in conclusion we recommend using partitioning tree based pattern spectra, and more specifically the ones calculated from the $(\omega)$-tree for soil structure analysis. 


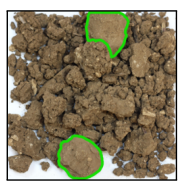

(a)

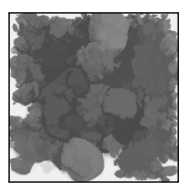

(b)

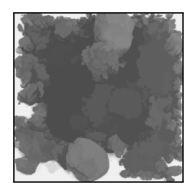

(c)

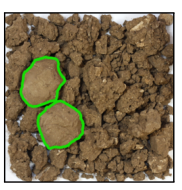

(d)

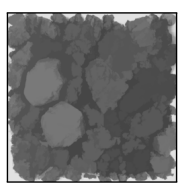

(e)

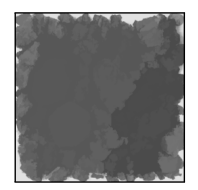

(f)

Fig. 5. Interaction of the tree of shapes with the aggregates positioned at the border with the background: (a) and (d) two different arrangements of soil A at close scale $\left(15 \times 15 \mathrm{~cm}^{2}\right)$ with two largest aggregates outlined in green, (b) and (e) filtering with area $200000 \mathrm{px},(\mathrm{b})$ and (e) filtering with area $600000 \mathrm{px}$. The aggregates are correctly removed in $(\mathrm{d})$ where they are positioned in the middle of the image, but not in (a) when bordering the background.

\section{Conclusions and Future Work}

We have compared the suitability of pattern spectra calculated from a collection of component trees for soil structure analysis, framed as an image retrieval problem, as well as the influence of different measures of removed image detail and histogram distances. We specifically examine the pattern spectra based on partitioning trees, self-dual hierarchies able to represent image regions of intermediate grey levels. Our experiments are designed to work with small sample sizes, and examine the influence of scale and visible background. We confirm the stable performance of the partitioning trees, and find that pattern spectra calculated from the $(\omega)$-tree using $\mathcal{M}_{\text {area }}$ and compared with the $L_{1}$ distance result in the best and most stable performance on the examined retrieval problem.

As part of future work, we plan to further investigate the behaviour of partitioning trees when sample sizes at multiple scales are considered. Some techniques towards possible performance improvement include fully removing the background before tree construction and working on images with partial support, as well as trying different size attributes which would more accurately reflect particles passing through differently sized sieve meshes. The final goal is correlating the image-based granulometry to the granulometry obtained through

physical measuring in a regression setup, as well as improving the robustness of the process for in-field deployment as opposed to working in laboratory setting.

\section{References}

1. Beare, M.H., Bruce, R.R.: A comparison of methods for measuring water-stable aggregates: implications for determining environmental effects on soil structure. Geoderma 56(1-4), 87-104 (1993)

2. Bianconi, F., Di Maria, F., Micale, C., Fernández, A., Harvey, R.: Grain-size assessment of fine and coarse aggregates through bipolar area morphology. Machine Vision and Applications 26(6), 775-789 (2015)

3. Bosilj, P., Aptoula, E., Lefèvre, S., Kijak, E.: Retrieval of remote sensing images with pattern spectra descriptors. ISPRS International Journal of Geo-Information 5(12), 228 (2016) 
4. Bosilj, P., Damodaran, B.B., Aptoula, E., Dalla Mura, M., Lefèvre, S.: Attribute profiles from partitioning trees. In: ISMM. pp. 381-392. Springer (2017)

5. Breen, E.J., Jones, R.: Attribute openings, thinnings, and granulometries. Computer Vision and Image Understanding 64(3), 377-389 (1996)

6. Bronick, C.J., Lal, R.: Soil structure and management: a review. Geoderma 124(12), 3-22 (2005)

7. Buscombe, D., Masselink, G.: Grain-size information from the statistical properties of digital images of sediment. Sedimentology 56(2), 421-438 (2009)

8. Cavallaro, G., Falco, N., Dalla Mura, M., Benediktsson, J.: Automatic attribute profiles. IEEE T. Image Process. 26(4), 1859-1872 (2017)

9. Cousty, J., Najman, L., Kenmochi, Y., Guimarães, S.: Hierarchical segmentations with graphs: quasi-flat zones, minimum spanning trees, and saliency maps. J Math Imaging Vis 60(4), 479-502 (2018)

10. Doulamis, A., Doulamis, N., Maragos, P.: Generalized multiscale connected operators with applications to granulometric image analysis. In: ICIP. vol. 3, pp. 684-687. IEEE (2001)

11. Frančišković-Bilinski, S., Bilinski, H., Vdović, N., Balagurunathan, Y., Dougherty, E.R.: Application of image-based granulometry to siliceous and calcareous estuarine and marine sediments. Estuarine, Coastal and Shelf Science 58(2), 227-239 (2003)

12. Graham, D.J., Reid, I., Rice, S.P.: Automated sizing of coarse-grained sediments: image-processing procedures. Mathematical Geology 37(1), 1-28 (2005)

13. Guimarães, R.M.L., Ball, B.C., Tormena, C.A.: Improvements in the visual evaluation of soil structure. Soil Use and Management 27(3), 395-403 (2011)

14. Manning, C.D., Raghavan, P., Schütze, H.: Introduction to Information Retrieval. Cambridge University Press (2008)

15. Maragos, P.: Pattern spectrum and multiscale shape representation. IEEE T Pattern Anal. 11(7), 701-716 (1989)

16. Matheron, G.: Random sets and integral geometry. Wiley New York (1975)

17. Monasse, P., Guichard, F.: Scale-space from a level lines tree. Journal of Visual Communication and Image Representation 11(2), 224-236 (2000)

18. Mora, C.F., Kwan, A.K.H., Chan, H.C.: Particle size distribution analysis of coarse aggregate using digital image processing. Cement and Concrete Research 28(6), 921-932 (1998)

19. Mueller, L., Kay, B.D., Hu, C., Li, Y., Schindler, U., Behrendt, A., Shepherd, T.G., Ball, B.C.: Visual assessment of soil structure: Evaluation of methodologies on sites in canada, china and germany: Part i: Comparing visual methods and linking them with soil physical data and grain yield of cereals. Soil and Tillage Research 103(1), 178-187 (2009)

20. Pina, P., Lira, C., Lousada, M.: In-situ computation of granulometries of sedimentary grains-some preliminary results. J. Coast. Res 64, 1727-1730 (2011)

21. Salehizadeh, M., Sadeghi, M.T.: Size distribution estimation of stone fragments via digital image processing. In: International Symposium on Visual Computing. pp. 329-338. Springer (2010)

22. Salembier, P., Oliveras, A., Garrido, L.: Anti-extensive connected operators for image and sequence processing. IEEE T. Image Process. 7(4), 555-570 (1998)

23. Soille, P.: On genuine connectivity relations based on logical predicates. In: ICIAP. pp. 487-492. IEEE (2007)

24. Soille, P.: Constrained connectivity for hierarchical image partitioning and simplification. IEEE T Pattern Anal. 30(7), 1132-1145 (2008) 
25. Urbach, E.R., Roerdink, J.B.T.M., Wilkinson, M.H.F.: Connected shape-size pattern spectra for rotation and scale-invariant classification of gray-scale images. IEEE T Pattern Anal. 29(2), 272-285 (2007)

26. Urbach, E.R., Wilkinson, M.H.F.: Shape-only granulometries and grey-scale shape filters. In: ISMM. pp. 305-314 (2002) 